\title{
QUALIDADE DE VIDA DE DISCENTES DO CURSO DE ENFERMAGEM
}

\author{
Raquel Vilanova Araujo ${ }^{1}$ \\ Aline Alves Cardoso -Centro Universitário Santo Agostinho (UNIFSA) ${ }^{2}$ \\ Bruna Larissa Fernandes Pereira -Centro Universitário Santo Agostinho (UNIFSA) ${ }^{2}$ \\ Regina Celia Vilanova Campelo -Universidade Federal do Piauí (UFPI) ${ }^{3}$ \\ Inez Sampaio Nery -Universidade Federal do Piauí (UFPI) ${ }^{4}$ \\ Ana Fátima Carvalho Fernandes- Universidade Federal do Ceará (UFC) ${ }^{5}$ \\ Viriato Campelo-Universidade Federal do Piauí (UFPI) ${ }^{6}$
}

\section{RESUMO}

Avaliar a qualidade de vida de discentes é importante para conhecer realidades e fragilidades destes, a fim de buscar estratégias para ajudá-los no enfrentamento e na melhoria da qualidade de vida, uma vez que o discente, como futuro enfermeiro, precisa ter equilíbrio quanto às condições físicas, mentais e emocionais para cuidar do próximo. Logo, objetivou-se avaliar os fatores preditores e a qualidade de vida de discentes do curso de Enfermagem. Trata- se de revisão integrativa de literatura, cuja busca de dados foi realizada nas bases de dados da Literatura Latino-Americana e do Caribe em Ciências de Saúde (LILACS) e Banco de Dados em Enfermagem (BDENF), com os descritores: estudantes de enfermagem, qualidade de vida, estudantes de enfermagem. Incluíram-se artigos em português, inglês e espanhol; e excluíramse estudos que não respondiam à pergunta da pesquisa, teses, dissertação e revisão. A amostra inicial foi de 49 estudos, após critérios de exclusão, ao final, obtiveram-se 17 para leitura, análise e categorização. Emergiram-se duas categorias: Conceito e avaliação da qualidade de vida: percepções de discentes do curso de Enfermagem; Fatores relacionados à qualidade de vida: percepções de discentes do curso de Enfermagem. Percebeu-se que os alunos do curso de Enfermagem, no decorrer da vida acadêmica, tendem a ter alterações na qualidade de vida, o que pode acarretar elevação do nível de estresse e ansiedade. Reconheceu-se a importância do apoio familiar e da ajuda de professores. O docente deve ter senso crítico, reflexivo e holístico em relação ao aluno, e buscar estratégias para ajudá-lo nesse processo, sendo fundamental o trabalho interdisciplinar.

Palavras-chave: Qualidade de Vida. Enfermagem. Estudantes de Enfermagem.

\footnotetext{
${ }^{1}$ Mestre em Ciências e Saúde - Universidade Federal do Piauí (UFPI), Docente do Curso de Graduação em Enfermagem - Centro Universitário Santo Agostinho (UNIFSA), E-mail: raquelvila@outlook.com. ${ }^{2}$ Enfermeira - Centro Universitário Santo Agostinho (UNIFSA), ${ }^{3}$ Doutora em Saúde Coletiva - Faculdade de Medicina da Universidade de São Paulo - USP, Docente - Universidade Federal do Piauí (UFPI), E-mail: regina.vilanova-campelo@usp.br, ${ }^{4}$ Doutora em Enfermagem, Docente - Universidade Federal do PiauíUFPI, ${ }^{5}$ Doutora em Enfermagem, Docente - Universidade Federal do Ceará-UFC, ${ }^{6}$ Doutor em Medicina, Docente - Universidade Federal do Piauí (UFPI).
} 
ANAIS CBCS 2019 | 3 a 5 de outubro de 2019 | Centro Universitário Santo Agostinho - Teresina - PI

\section{INTRODUÇÃO}

Qualidade de vida é um termo de difícil definição, pois envolve variedade de condições que afetam a percepção de indivíduos, sentimentos e comportamentos relacionados à condição de saúde, abrangendo significados que refletem conhecimentos, experiências e valores de um indivíduo ou coletividade em várias épocas, espaços e histórias e que se encontram em construção social e cultural (MACHADO; BERGMANN, 2012; MINAYO et al., 2000).

A qualidade de vida pode ser abordada de forma subjetiva, quando se analisam o bem-estar, a satisfação, as relações sociais e ambientais e culturais do indivíduo; e de forma objetiva, quando se avaliam as condições de educação, o transporte, a moradia e o trabalho (ALMEIDA et al., 2012).

De acordo com Almeida et al. (2012), a Qualidade de vida está relacionada às condições de vida de um ser humano e envolve o bem-estar físico, mental, psicológico, emocional, social, assim como a saúde, a educação e outras circunstâncias de vida. Marques (2015) completa que há íntima relação com os aspectos socioeconômicos de determinada população, as condições e o modo de vida dos sujeitos. Cada indivíduo tem percepção diferente sobre qualidade de vida, o que varia conforme o contexto da cultura e o sistema de valores no qual ele vive e na relação com os próprios objetivos, expectativas, padrões e preocupações (GESTEIRA, 2016).

Gesteira (2016) completa afirmando que a qualidade de vida envolve o bemestar espiritual, físico, mental, psicológico e emocional, além de relacionamentos sociais, como família e amigos e, também, saúde, educação, habitação saneamento básico e outras circunstâncias da vida, podendo ser afetada por problemas familiares, financeiros, no trabalho, estudo, dentre outras.

Para o indivíduo que inicia a formação superior, exigem-se responsabilidade, compromisso, dedicação, além de cobranças quanto ao desempenho, à formação profissional, à preocupação com mercado de trabalho e ao salário. Além da sobrecarga de tarefas, como o trabalho, as atividades domésticas, a família/filhos, os problemas financeiros que trazem repercussões negativas na qualidade de vida de discentes, que

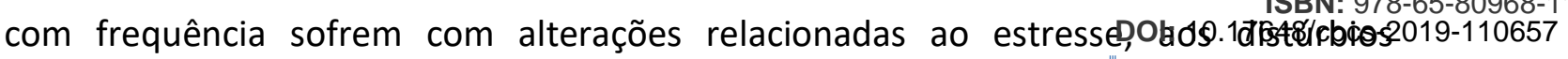


ANAIS CBCS 2019 | 3 a 5 de outubro de 2019 | Centro Universitário Santo Agostinho - Teresina - P emocionais e psíquicos (GOLVEIA et al., 2017).

Tassini et al. (2017) completam que a graduação implica grandes mudanças que podem influenciar a vida de estudantes, de forma positiva ou negativa, a qualidade de vida destes. Durante o curso, o discente perpassa por situações que necessitam de equilíbrio emocional. Os fatores que ocasionam ansiedade nos discentes, em geral, são decorrentes de perspectivas e dúvidas em relação ao futuro acadêmico, expectativas familiares, problemas financeiros (MARCHI et al., 2013). O estresse é outro problema frequente em alunos e influencia diretamente no desenvolvimento de atividades acadêmicas. Quando não controlado, pode comprometer a saúde mental, emocional e o desempenho profissional (SILVA et al. 2016).

Diante dessa situação, é fundamental direcionar atenção aos discentes que adentram nos cursos de graduação, uma vez que estes estarão lidando com seres humanos, tendo contato com sofrimento de outras pessoas, sendo, assim, essencial que este tenha total equilíbrio das condições emocionais e psíquicas para lidar com o próximo (MARCHI et al., 2013). O autor citado afirma que o discente que sofre de ansiedade tem o aprendizado diminuído, o que acarreta danos no desempenho acadêmico.

De acordo com Moura et al. (2016), é fundamental que instituições de ensino realizem discussões sobre o processo de formação de cuidadores de Enfermagem, a fim de se adaptarem às diversas fases da vida acadêmica. Benavente et al. (2014) defendem que é necessário que se crie instrumento de suporte para esses alunos, para que estes percebam o apoio da instituição de ensino. Esse auxílio é necessário para ajudar os discentes no processo de enfrentamento dos problemas e do sofrimento (BENAVENTE et al., 2014). As discussões relacionadas ao tema são relevantes, pois os discentes devem ter consciência acerca da importância da manutenção da boa condição de vida e saúde, para que possa prestar cuidado de qualidade e com segurança ao próximo.

Assim, em linhas gerais, objetivou-se conhecer a qualidade de vida de discentes do curso de Enfermagem; e, especificamente, descrever os domínios mais comprometidos da qualidade de vida e identificar os fatores preditores relacionados à qualidade de vida dos discentes investigados. 
ANAIS CBCS 2019 | 3 a 5 de outubro de 2019 | Centro Universitário Santo Agostinho - Teresina - P

Trata-se de revisão integrativa de literatura que percorreu as etapas: elaboração

da questão de pesquisa; busca na literatura dos estudos primários; extração de dados; avaliação dos estudos primários; análise e síntese dos resultados; e apresentação da revisão (GALVÃO; MENDES; SILVEIRA, 2010). As questões norteadoras da revisão integrativa foram: omo é a qualidade de vida de discentes do curso de enfermagem? Quais os fatores preditores relacionados? Para responder às pergunta da pesquisa, utilizaram-se da estratégia PICo, onde o P diz respeito à população (Discentes); I, o Interesse (fatores preditores e qualidade de vida); Co, o contexto (curso de enfermagem).

Realizou-se a busca dos estudos primários nas bases de dados da Literatura Latino-Americana e do Caribe em Ciências de Saúde (LILACS) e Banco de Dados em Enfermagem (BDENF), utilizando-se dos descritores controlado sem Ciências da Saúde (DeCS): estudantes de enfermagem, qualidade de vida. Incluíram-se estudos primários que retratassem a qualidade de vida de discentes do curso de enfermagem e os fatores preditores relacionados à qualidade de vida, artigos disponíveis na íntegra; e excluídos, artigos de revisão da literatura, duplicados, teses, dissertações e manuais.

A amostra inicial foi composta de 127 artigos, após aplicabilidade dos critérios de inclusão e exclusão, restaram 49 estudos primários, após leitura de títulos e resumos, identificou-se amostra final de 17 estudos primários para análise e discussão. A coleta de dados dos estudos primários incluídos na revisão integrativa foi realizada com auxílio de instrumento elaborado pelos pesquisadores que consta de informações relacionadas ao sujeito da pesquisa, metodologia, tamanho da amostra, mensuração de variáveis, instrumento utilizado, método de análise e conceitos embasadores empregados.

A análise de dados foi realizada em cinco etapas, conforme descrito por Moraes, (1999): preparação das informações, unitarização ou transformação do conteúdo em unidades, categorização ou classificação das unidades em categorias, descrição e interpretação. Construíram duas categorias temáticas: Conceito e avaliação da qualidade de vida: percepções de discentes do curso de Enfermagem; Fatores relacionados à qualidade de vida: percepções de discentes do curso de Enfermagem. 


\section{congęESSO CIENCIAESOCIEDADE \\ Inovação, Diversidaale e Sustentahilitilade}

ANAIS CBCS 2019 | 3 a 5 de outubro de 2019 | Centro Universitário Santo Agostinho - Teresina - PI

\section{Conceito e avaliação da qualidade de vida: percepções de discentes do curso de Enfermagem}

Na percepção dos alunos, a qualidade de vida foi considerada equilíbrio entre a vida profissional e pessoal (PEREIRA; PINHO; CORTE, 2016). Nos estudos analisados, percebeu-se que os acadêmicos de enfermagem avaliaram a qualidade de vida satisfatória e satisfação com a própria saúde.

Pereira, Pinho e Corte (2016) destacam que a saúde depende de fatores como alimentação, renda, educação, moradia e o próprio emocional. Nos estudos analisados, observou-se que os alunos dos semestres iniciais passavam por adaptação na vida, mudança necessária devido às exigências da vida acadêmica que requer tempo e determinação dos alunos. De acordo com Gama (2016), a adaptação em relação à carga horária de estudo, às responsabilidades com tarefas da faculdade, aos trabalhos e às provas pode comprometer o domínio social da qualidade de vida.

Observou-se que o domínio físico da qualidade de vida dos discente foi mais comprometido devido a os esforços físicos das tarefas do cotidiano, os procedimentos realizados nos estágios e de demandas extracurriculares. O domínio psicológico foi o melhor avaliado pelos alunos do curso de Enfermagem, o que foi explicado pelo fato de se apoiarem nas crenças pessoais, boa autoestima, aceitação da aparência física e dos sentimentos positivos (SILVA et al., 2013). No domínio relações sociais, os alunos entrevistados relataram que estavam satisfeitos igualmente quanto ao relacionamento e apoio de amigos e familiares. De acordo com Gama (2016), para que os alunos tenham bom desempenho em tarefas, é necessário que tenham uma rede de apoio dos familiares, amigos ou pessoas que vivem com eles, para poderem ter boa qualidade de vida.

O domínio físico foi avaliado por algumas questões, como sono, capacidade de locomoção, energia para o dia a dia, capacidade de trabalhar, disposição diante da dor, capacidade de desempenhar tarefas e não dependência de tratamentos. As dificuldades de locomoção, a dor e a dependência de tratamentos foram relacionadas ao comprometimento do domínio físico da qualidade de vida.

O domínio meio ambiente que é composto por oito facetas: atividades de lazer,

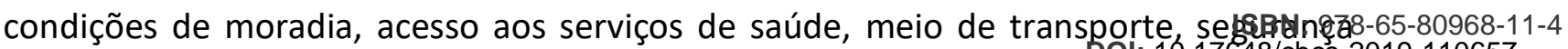




\section{CONGEESSOOCIENCIAESOCIEDADE

ANAIS CBCS 2019 | 3 a 5 de outubro de 2019 | Centro Universitário Santo Agostinho - Teresina - PI física, ambiente físico, recurso financeiros e oportunidades de novas informações foi o pior avaliado. $\mathrm{O}$ acesso ao serviço de saúde, as condições de moradia, o ambiente físico e as oportunidades de novas informações apresentaram avaliação insatisfatória.

\section{Fatores relacionados à qualidade de vida: percepções dos discentes do curso de Enfermagem}

Os alunos associaram a qualidade de vida e a satisfação das necessidades humanas com o ter saúde, moradia, família e acesso a meios de transporte de qualidade. Para eles, o cansaço, o sono, as exigência do curso, a carga horária extensa, os plantões, as atividades extracurriculares geram esgotamento físico e mental intenso e comprometem de forma negativa a qualidade de vida dos alunos.

Os fatores que favoreceram a qualidade de vida foram: interação com as pessoas, e as amizades conquistadas, a construção de conhecimento e a ampliação da visão de mundo. Os estudantes citaram alguns fatores que comprometeram a qualidade de vida, como aulas pouco didáticas, estágios exigentes, ausência de ética com os alunos (PEREIRA; PINHO; CORTEZ, 2016).

Para os estudantes, os fatores que influenciaram negativamente na qualidade de vida foram sono, capacidade para desempenhar as tarefas do cotidiano, recursos financeiros e oportunidade de lazer, os quais conduziram para o pior desempenho acadêmico.

Esses fatores estão ligados diretamente com o desempenho de tarefas e aprendizado, pois caso algum deles estejam insatisfatório, poderá causar danos para qualidade de vida. Brigitt (2017) revelou que dentre os motivos que desencadeavam alterações no nível de ansiedade, o principal era o Trabalho de Conclusão de Curso, além das atividades de estágio curricular e estágio não obrigatório.

Nos estudos analisados, observou-se que a pouca disponibilidade de tempo para estar com a família, parentes e amigos ou realizar atividades de lazer acarreta para o estudante carga de estresse e desgaste emocional e físico. Em estudo realizado em universitários de instituição pública se constatou prevalência de depressão em 30,2\% dos alunos e de ansiedade, em 62,9\%. Identificou-se associação entre o nível de sintomas depressivos, trabalho, sexo e lazer (FERNANDES et al., 2018). 


\section{CONGQEESSOCIENCIAESOCIEDADE

ANAIS CBCS 2019 | 3 a 5 de outubro de 2019 | Centro Universitário Santo Agostinho - Teresina - PI

De acordo com Carolina (2018), algumas situações são geradores de estresse,

como tempo e lazer, a falta de tempo e lazer para descansar tem sido a principal causa de estresse entre universitários. Além disso, o discente, ao iniciar as atividades práticas, sente-se despreparado, pois espera-se que consiga realizar as práticas, de acordo com o que foi aprendido na teoria, nessa ocasião, depara-se com momento de tensão e preocupação, gerando, assim, insegurança diante das novas situações que requerem a habilidade e experiência dos alunos.

De acordo com Benavente et al. (2014), o estresse para o acadêmico altera antes e após o estágio, mostram resultados que indicam que os alunos estão bastante estressados, tendo os sintomas fisiológicos e emocionais, como diarreia, angústia, aumento da sudorese, cansaço constante, irritabilidade, insônia e mudança de hábito alimentar. Alterações positivas ou negativas podem acarretar um nível de estresse.

Explicam-se, ainda, a satisfação e insatisfação de estudantes quanto à capacidade física, o que pode alterar o aprendizado destes. E alguns alunos afirmam não se sentir influenciados por fatores externos, como sentimentos negativos, autoestima, imagem corporal e religião. A qualidade de vida de estudantes diminuiu a partir do segundo ano de curso, com a inclusão de alunos em campos hospitalares, pois terão que mostrar habilidade teórica, através dos estágios supervisionados, nas disciplinas específicas para cada prática (PEREIRA et al., 2016).

Moura et al. (2016) observaram os acadêmicos, e o maior escore de qualidade de vida foi para o domínio físico; e o menor, para o domínio meio ambiente.

\section{CONCLUSÕES}

Diversos fatores podem afetar a qualidade de vida de discentes, como as condições sociais, econômicas, suporte familiar/amigos e as atividades acadêmicas. 0 domínio mais comprometido foi o meio ambiente (condições de moradia, lazer, recursos financeiros, segurança física etc).

Nos discentes, observou-se com frequência estresse e ansiedade, em geral ocasionados pelas atividades curriculares e extracurriculares, pouca disponibilidade de tempo para atividades de lazer, ficar com família e amigos e descansar. É fundamentantal 978-65-80968-11-4 
ANAIS CBCS 2019 | 3 a 5 de outubro de 2019 | Centro Universitário Santo Agostinho - Teresina - P

o fortalecimento das redes de apoio de instituições de ensino, entidades governamentais, professores, familiares e amigos para apoiar discentes no processo de enfrentamento das dificuldades relacionadas à vida acadêmica que, muitas vezes, é impactada por conflitos em outras áreas. O docente deve ter senso crítico, reflexivo e holístico em relação ao aluno, e buscar estratégias para ajudá-lo nesse processo, sendo fundamental o trabalho interdisciplinar.

\section{REFERÊNCIAS [Todas foram corrigidas]}

ALMEIDA, M. A. B.; GUTIERREZ, G. L.; MARQUES, R. Qualidade de vida, definições, conceitos e interfaces com outras áreas de pesquisa. São Paulo: Escola de Artes, Ciências e Humanidades EACHE/USP, 2012. Disponível em:<http://each.uspnet.usp.br/edicoes-each/qualidade_vida.pdf>. Acesso em: 23 jul. 2019.

BENAVENT, S. B. T. et al. Influência de fatores de estresse e características sociodemográficas na qualidade do sono de estudantes de enfermagem. Rev Escola Enferm USP, v.48, n. 3, p. 514-519, 2014. Disponível em: http://www.scielo.br/pdf/reeusp/v48n3/pt_0080-6234-reeusp- 48-03-514.pdf. Acesso em: 20 fev. 2019.

CUNHA, M. Bem-estar em estudantes do ensino superior. Educ Desenv Social, série 2, n. 2, edição especial, 2017. Disponível em:

<https://revistas.rcaap.pt/millenium/article/view/12098>. Acesso em: 21 abr. 2019

FERNANDES, M. A. et al. Prevalência de sintomas ansiosos e depressivos em universitários de uma instituição pública. Rev Bras Enferm., v. 71, supl. 5, p. 2169-2175,

$$
\text { 2018. Disponível em }
$$

<http://www.scielo.br/scielo.php?script=sci_arttext\&pid=S0034-

71672018001102169\&lng=pt\&nrm=iso>. Acesso em: 08 set. 2019.

GOUVEIA, M. T. O. et al. Qualidade de vida e bem-estar dos estudantes universitários de enfermagem: revisão integrativa. Rev Enferm UFPI, v. 6, n. 3, p. 72-78, 2017. Disponível em:

$<$ http://bases.bireme.br/cgi-

bin/wxislind.exe/iah/online/?IsisScript=iah/iah.xis\&src=google\&base=BDENF\&lang=p\& nextAction=Ink\&exprSearch=32003\&indexSearch=ID>. Acesso em: 29 fev. 2019.

MARCHI, K. C. et al. Ansiedade e consumo de ansiolíticos entre estudantes de enfermagem de uma universidade pública. Rev Eletr Enferm., v. 15, n. 3, p. 731-739, 2013. Disponível em:

<https://www.fen.ufg.br/revista/v15/n3/pdf/v15n3a15.pdf.> Acesso em: 20 fevS.g0199.8-65-80968-11-4 DOi: $10.17648 / c b c s-2019-110657$ 


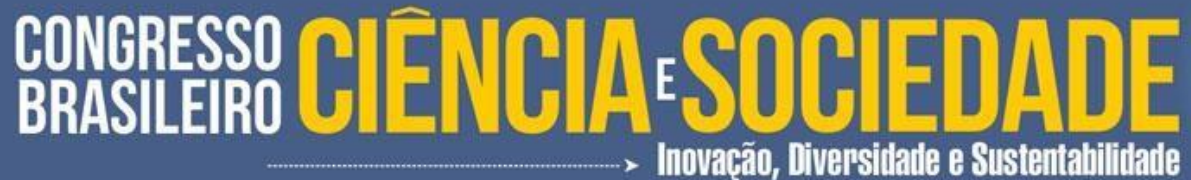

ANAIS CBCS 2019 | 3 a 5 de outubro de 2019 | Centro Universitário Santo Agostinho - Teresina - P

MARQUES, R. F. R. Qualidade de vida, atividade física e saúde: relações na busca de uma vida melhor [Internet]. Disponível em:

<biblioteca.universia.net/...qualidadevidautilidade...populaçãoporto/.../5..>. Acesso em: 13 jan. 2019.

MINAYO, M. C. S.; HARTZ, Z. M. A.; BUSS, P. M. Qualidade de vida e saúde: um debate necessário. Ciênc Saúde Coletiva, v. 5, n. 1, p. 7-18, 2000 . Disponível em:

<http://www.scielo.br/scielo.php?script=sci_arttext\&pid=S1413-

$81232000000100002 \&$ Ing=en\&nrm=iso $>$. Acesso em: 08 set. 2019.

MOURA, I. H. et al. Qualidade de vida de estudantes de graduação em enfermagem. Rev

Gaúcha Enferm., v. 37, n. 2, e55291, 2016.

Disponível em:

<http://www.scielo.br/scielo.php?script=sci_arttext\&pid=S1983-

14472016000200407\&Ing=en\&nrm=iso>. Acesso em: 08 set. 2019.

PEREIRA, M. O.; PINHO, P. H.; CORTES, J. M. Qualidade de vida: percepção de discentes de graduação em enfermagem. J Nurs Health, v.6, n.2, p.321-333, 2016. Disponível em:

<https://periodicos.ufpel.edu.br/ojs2/index.php/enfermagem/article/view/5780> Acesso em: 20 fev. 2019.

SILVA, A. R. S. et al. Estudo do estresse na graduação de enfermagem: revisão integrativa de literatura. Semina Cienc Biol Saúde., v. 2, n. 3, p. 75-86, 2016. Disponível em: <https://periodicos.set.edu.br/index.php/facipesaude/article/view/3211/2082> Acesso em: 21 fev. 2019.

TASSINI, C. C. et al. Avaliação do estilo de vida em discentes universitários da área da saúde através do questionário fantástico. Int J Cardiovas Sci., v. 30, n. 2, p. 117-122, 2017.

Disponível

em:

<http://www.scielo.br/scielo.php?script=sci_arttext\&pid=S2359-

56472017000200117\&lng=en\&nrm=iso>. Acesso em: 08 set. 2019. 